\title{
Solar energetic particle event onset as analyzed from simulated data
}

\author{
J. Lintunen and R. Vainio \\ Department of Physical Sciences, PO Box 64, 00014 University of Helsinki, Finland \\ Received 1 September 2003 / Accepted 25 February 2004

\begin{abstract}
Solar energetic particle (SEP) event onset is analyzed using simulated data. A large number of simulations using different coronal and interplanetary (IP) scattering conditions are performed. Protons in the energy range of 0.13-57 MeV are considered. The simulated data are analyzed employing a velocity dispersion analysis (VDA) to the proton-flux onset times in 16 energy channels. As a result of the analysis, the apparent coronal release time, $t_{0}$, and the apparent IP path length, $s$, of the first-observed particles are obtained. It is shown that typical IP scattering conditions, i.e., 1-GV radial mean free path of $\Lambda_{r r}=0.1-1$ AU with a $\propto P^{1 / 3}$ rigidity dependence, lead to apparent path lengths of $s \sim 1-2 \mathrm{AU}$, consistent with observations. Thus, SEP events with $s \sim 2$ AU can simply be explained as a result of IP scattering. Models with coronal mean free paths small enough to enable proton acceleration to high energies in quasi-parallel shocks close to the Sun are shown to yield VDA results in agreement with observations, at least with $\Lambda_{r r} \gtrsim 0.3$. As a result of IP scattering, the inaccuracy of the release times is tens of minutes in events with $s \gtrsim 2 \mathrm{AU}$, which may prevent accurate timing of particle release using the VDA method in such events. Low contrast between the time-of-maximum intensity of the studied SEP event and the pre-event background intensity and/or a large difference between the forms of the respective energy spectra may also lead to errors in derived onset times. Best timing results (with errors typically less than $10 \mathrm{~min}$ ) are obtained for large IP mean free paths, $\Lambda_{r r} \gtrsim 0.3 \mathrm{AU}$ (typically resulting in small values of $s \lessgtr 1.5 \mathrm{AU}$ ), and large intensity contrast between the SEP event and the pre-event background.
\end{abstract}

Key words. Sun: particle emission - Sun: coronal mass ejections (CMEs) - Sun: flares - Sun: solar wind - turbulence

\section{Introduction}

One of the open questions of solar energetic particle (SEP) research is the origin of accelerated particles in SEP events. While shocks driven by coronal mass ejections (CMEs) and flares are thought to be the sites of solar particle acceleration (see, e.g., the review by Reames 1999), the details of the acceleration processes are still uncertain: it is not clear where, when or how the CME-driven shocks most efficiently accelerate particles in gradual SEP events, nor is it known what the flare-related acceleration mechanisms are operating in impulsive SEP events.

One of the observational methods to obtain information on the details of the acceleration process is to analyze timing of the SEP events. In these studies, one determines the SEP-event onset time, $t_{\text {onset }}$, in several energy channels, $E_{j}$, for one or more particle species. In the simplest version of the subsequent analysis (e.g., Kahler 1994; Klein et al. 1999), one assumes that the path length $s$ traveled by the particles after their release from the acceleration site in all energy channels corresponds to a pre-defined distance $(\sim 1 \mathrm{AU})$ along the magnetic field line connecting the acceleration region to the observer. This enables one to determine the release time of

Send offprint requests to: R. Vainio,

e-mail: rami.vainio@helsinki.fi particles from the acceleration site at different energies. We call this method "solar release time analysis" (RTA). Another version of the analysis (e.g., Torsti et al. 1998; Krucker \& Lin 2000) assumes the release time, $t_{0}$, and path length, $s$, to be independent of energy, but makes no assumptions on their values. The parameters are determined by fitting a straight line to the points $\left\{1 / v\left(E_{j}\right), t_{\text {onset }}\left(E_{j}\right)\right\}$, where $v(E)$ is the speed of the particle of energy $E$ :

$t_{\text {onset }}(E)=t_{0}+s / v(E)$.

We call this method "velocity dispersion analysis" (VDA). Both models allow a comparison of the determined release time(s) to the timing of different electromagnetic emissions, while the latter also provides some information on the geometry and/or particle transport conditions in the interplanetary (IP) medium.

Recently, there has been strong interest to apply VDA to determine the solar release time and, consequently, the solar processes responsible for particle acceleration at the Sun. Tylka et al. (2003) analyze two large impulsive events and three ground-level enhancements (GLEs) and deduce that impulsive event onset coincides with X-ray emission, whereas the GLE onset is delayed, implying a flare and CME shock origin for the two types of events. Mewaldt et al. (2003) analyze heavy-ion and electron fluxes in 11 events and find that heavy 
ions are generally released later than electrons, and that there is a correlation between the delay of the heavy-ion release times and the ${ }^{3} \mathrm{He}$-to- ${ }^{4} \mathrm{He}$ ratio consistent with the same scenario. Posner \& Kunow (2003) analyze one event, which seems not to be well fitted by a single value for $s$, but that low-energy observations seem to require a larger value for this parameter than high-energy observations; they suggest this to be due to a rigidity dependent SEP scattering in the IP medium. All the recent studies, as well as the earlier ones (e.g., Torsti et al. 1998; Krucker \& Lin 2000), give values of $s$ that typically fall in the range of $s \approx 1-2 \mathrm{AU}$.

Existing studies on SEP-event onset usually do not provide rigid theoretical justification of the assumptions underlying their analysis. It is well known that the mean free path, $\lambda$, of protons related to their scattering off the magnetic turbulence in the IP medium can be much smaller than $1 \mathrm{AU}$. Typical values of the mean free path were estimated by Palmer (1982) to lie in the range of $\lambda=0.08-0.3 \mathrm{AU}$ in near Earth space in a wide range of rigidities around $100 \mathrm{MeV}$. More recent estimates (see, e.g., Sect. 8.1 of Reames 1999) yield values that are larger than this. Scattering should have a strong influence on the proton arrival times, at least when $\lambda$ is near the smaller extreme of the typical range. In addition, to have favorable conditions for CME shock acceleration, the coronal mean free path cannot be larger than a fraction of the local radial distance from the Sun (Vainio et al. 2000) if the shock propagates quasi-parallel to the magnetic field; for a quasi-perpendicular shock geometry this condition is more relaxed (e.g., Vainio \& Khan 2004). Diffusion through such a turbulent coronal region should add to the path length between the acceleration and observation. Furthermore, the mean free path (at least at $1 \mathrm{AU}$ ) is known to depend on the proton speed, which complicates the interpretation of the analysis results.

The purpose of this paper is to study the onset of SEP events from simulated data. In addition to obtaining guidelines for the analysis of observational data, the analysis also gives us constraints on the IP scattering conditions that are consistent with the observations of event onset. Different scenarios of the coronal/IP transport conditions as well as the injection time profile are studied to determine the validity of the timing obtained by the simple observational estimates. We will concentrate on protons, since their transport in the IP medium is usually more influenced by scattering than that of electrons.

\section{Particle transport model}

The model of focused transport of SEPs including the solar wind effects of convection and adiabatic deceleration (Ruffolo 1995 ) is used. Our simulations are based on the Monte Carlo method (Kocharov et al. 1998): individual particles are released impulsively from the acceleration site placed close to Sun and traced in the guiding-center approximation. We take the radial solar wind speed to be $V_{r}=430 \mathrm{~km} \mathrm{~s}^{-1}$ throughout the corona and IP space and the background magnetic field to be a Parker spiral with the spiral angle $\psi$ calculated from $\tan \psi=\Omega_{\odot} r / V_{r}$, where $\Omega_{\odot}$, here taken as $3 \times 10^{-6} \mathrm{~s}^{-1}$, is the angular speed of solar rotation. To take account of the IP electric field, $\boldsymbol{E}=-\boldsymbol{V} \times$ $\boldsymbol{B}$, we follow the particle motion in the frame co-rotating with the Sun, where the flow is along the field lines and no electric field appears. In this frame, the solar wind has a speed of $V(r)=$ $V_{r} / \cos \psi(r)$.

Particles are moved in small time steps, $\Delta t$, along the magnetic field lines using $\Delta r=v \mu \cos \psi \Delta t$ to advance the radial coordinate of the particle. Here, $v$ and $\mu$ are the particle speed and pitch-angle cosine measured in the co-rotating frame. Since the magnetic field in this frame is static, $v$ is constant during this motion. Conservation of a particle's first adiabatic invariant, $\left(1-\mu^{2}\right) / B(r)$, however, leads to an increase of $\mu$ after each time step. (We define $\mu>0$ to be outward from the Sun.) Effects of centrifugal and Coriolis forces are neglected, which means that the model is valid only at clearly suprathermal energies ( $E \gg 1 \mathrm{keV}$ for protons). After each time step, the particles are also subject to isotropic small-angle scatterings off magnetic turbulence. Scatterings are performed by rotating the particle velocity vector to a randomly chosen direction, $\varphi$, by a small, randomly-chosen scattering angle, $\vartheta$, giving the new pitch-angle cosine as

$\mu \leftarrow \mu \cos \vartheta+\sqrt{1-\mu^{2}} \sin \vartheta \cos \varphi$

(Kocharov et al. 1998; Vainio et al. 2000). The scattering frequency, determining the variance $\left\langle\vartheta^{2}\right\rangle=2 v \Delta t$, is $v(r, v)=v / \lambda$, where the mean free path $\lambda(r, P)$, is a pre-defined parameter dependent on $r$ and on particle rigidity, $P$. (Note that Kocharov et al. (1998) and Vainio et al. (2000) used a definition of $v=v / 2 \lambda$, i.e., $\left\langle\vartheta^{2}\right\rangle=4 v \Delta t$.) The scattering centers are assumed to be frozen in into the solar wind. Thus, prior to each scattering, $v$ and $\mu$ are transformed to the scattering-center frame; the scattering is then performed using Eq. (2) and conserving $v$ in this frame; after the scattering, both $v$ and $\mu$ are transformed back to the co-rotating frame. This scattering model leads to convection of the particles with the scattering centers at $V$ along the field lines, as well as to a decrease of the average particle energy measured in the co-rotating frame, i.e., to adiabatic deceleration (Kocharov et al. 1998).

The mean free path is modeled by the following parameterizations:

$\lambda=\frac{\lambda_{r r}}{\cos ^{2} \psi}$

$\lambda_{r r}(r, P)=\Lambda_{r r}\left(\frac{P_{1}^{2}+P^{2}}{P^{2}}\right)^{\alpha / 3}\left(\frac{P}{P_{0}}\right)^{1 / 3} \frac{r^{2}}{r^{2}+a^{2}}$,

where $P_{0}=1 \mathrm{GV}, P_{1}=50 \mathrm{MV}$, and $\Lambda_{r r}$ and $\alpha$ are parameters fixing the magnitude and the (low-)rigidity dependence of the mean free path. Theoretical values of the latter vary a lot, because scattering of low-rigidity particles crucially depend on various corrections (e.g., Bieber et al. 1994; Schlickeiser \& Achatz 1993; Vainio 2000) made to the quasilinear theory of scattering. The radial dependence is determined by the parameter $a$, taking values $a^{2} \ll r_{1}^{2}$ with $r_{1}=1 \mathrm{AU}$. This allows us to consider cases with a spatially constant radial mean free path $\lambda_{r r}$ in the IP space combined with different levels of turbulence close to the Sun. Our parameterization of $\lambda_{r r}$ is mathematical and not based on any physical model of the underlying magnetic turbulence. Note also that Eq. (3) is valid only if diffusion of particles perpendicular to the field lines is neglected, 
as we have done in the present study. This may not always be a valid approximation in the case of SEP events related to solar eruptions originating far from the field line connected to the observer (Zhang et al. 2003a,b). Inclusion of cross-field diffusion is, however, beyond the scope of the present modeling.

Particles are recorded in a two-dimensional array with dimensions $(t, 1 / v)$ after each time step, if they are inside a small "detector volume", $r \in\left[r_{1}, r_{1}+\delta r\right]$, where $\delta r \ll r_{1}$. This array can be normalized to give particle intensities as a function of time and energy. We also count the particles in another array with the same dimensions, but now weighting each recorded particle with its $\mu$. Dividing the latter array by the former one gives us the first-order anisotropy, $\langle\mu\rangle$. In addition, a two dimensional array with dimensions $(\mu, 1 / v)$ is recorded, which gives a time-integrated pitch-angle distribution.

We consider a wide range of inverse speeds, $\beta^{-1} \equiv c / v \in$ $[3,60]$, corresponding to proton energies $E \in[0.13,57] \mathrm{MeV}$. Different forms of the injection spectrum can be studied by weighing the simulated particles according to their initial energy each time they are recorded.

\section{Simulated data set}

The SEP event onset is analyzed from the Monte Carlo simulated proton data. The $1-\mathrm{GV}$ radial mean free path at $1 \mathrm{AU}$ is varied in the range $\Lambda_{r r} \in[0.1,1.0]$ AU corresponding to typical observational results. Most simulations are performed with an impulsive release of particles at $r_{\mathrm{inj}}=1.5 r_{\odot}$, i.e., using the source function (in particles per unit time, unit energy, and unit radial distance)

$Q_{0}=\frac{\mathrm{d} N}{\mathrm{~d} E} \delta(t) \delta\left(r-r_{\mathrm{inj}}\right)$.

The energy spectrum is taken as $\mathrm{d} N / \mathrm{d} E \propto E^{-2}$. In addition to the direct analysis of the simulated data with impulsive injection, these data are used as "Green's functions," i.e., convoluted with temporally extended injection profiles to effectively solve the problem for the injection function

$Q_{1}=\frac{\mathrm{d} N}{\mathrm{~d} E} \frac{\mathcal{H}(\tau-t) \mathcal{H}(t)}{\tau} \delta\left(r-r_{\text {inj }}\right)$,

where $\mathcal{H}(t)$ is the step function and $\tau$ is a constant injection time scale. Hence, $Q_{1} \stackrel{\tau \rightarrow 0}{\longrightarrow} Q_{0}$.

We have performed a number of simulations to create a data base for further analysis of SEP event onsets under different IP scattering conditions. The input values for the simulation runs are listed in Table 1. Our aim is to perform a parametric study of the effect of the coronal/IP transport conditions on the validity of SEP onset analysis. For this purpose, we analyze the simulated data set using VDA. As a definition of the event onset time, $t_{\text {onset }}\left(E_{j}\right)$, in each energy channel, $E_{j}$, we use the time when the intensity first exceeds a fixed fraction $k$ of the maximum intensity in that channel, i.e.,

$t_{\text {onset }}\left(E_{j}\right)=\min \left\{t_{i} \mid I\left(t_{i}, E_{j}\right)>k I_{\max }\left(E_{j}\right)\right\}$

with the values of $k$ between 0.001 and 0.1 . Thus, the normalization of the energy spectrum in our analysis can be arbitrary, and different values of $k$ may simulate the strength of
Table 1. Input parameters for the simulation runs performed to study the onset of SEP events.

\begin{tabular}{cccc}
\hline \hline No. & $\Lambda_{r r}[\mathrm{AU}]$ & $\alpha$ & $a\left[r_{\odot}\right]$ \\
\hline 1 & 0.1 & 0 & 0 \\
2 & 0.2 & 0 & 0 \\
3 & 0.3 & 0 & 0 \\
4 & 0.4 & 0 & 0 \\
5 & 0.5 & 0 & 0 \\
6 & 0.6 & 0 & 0 \\
7 & 0.8 & 0 & 0 \\
8 & 1.0 & 0 & 0 \\
& & & \\
9 & 0.1 & 0 & 30 \\
10 & 0.3 & 0 & 30 \\
11 & 1.0 & 0 & 30 \\
& & & \\
12 & 0.1 & 1 & 0 \\
13 & 0.3 & 1 & 0 \\
14 & 1.0 & 1 & 0 \\
& & & \\
15 & 0.1 & 1 & 30 \\
16 & 0.3 & 1 & 30 \\
17 & 1.0 & 1 & 30 \\
\hline
\end{tabular}

the event relative to the pre-event conditions. The analysis is performed over the full set of energy channels $(0.13-57 \mathrm{MeV})$ used in the simulations, and separately over the low-energy (0.13-2.5 MeV) and high-energy (1-57 MeV) channels of the data set, corresponding to typical energy ranges in particle instruments using different measurement techniques. The energy channels are evenly spaced in $\beta^{-1}$, a total of 16 channels between $\beta^{-1} \in[3,60]$ are used, and the minimum value of $\beta_{j}^{-1}$ is used in each channel when fitting the onset data. The results of the VDA are given in Table 2. Throughout this paper, we use a $\mathrm{d} N / \mathrm{d} E \propto E^{-2}$ injection spectrum and normalize the simulated event so that the maximum intensities at $E=1 \mathrm{MeV}$ are $I\left(E_{0}\right)=10$ protons $\left(\mathrm{cm}^{2} \mathrm{~s} \mathrm{sr} \mathrm{MeV}\right)^{-1}$, corresponding to a medium-sized SEP event. An example of the simulated intensities and the resulting VDA is plotted in Fig. 1.

\section{Results and discussion}

Let us, first, consider the VDA fitting parameters as a function of proton mean free path. As a "standard scenario" we consider an impulsive particle injection at $r=1.5 r_{\odot}$ to an IP medium with a spatially constant mean free path with a rigidity dependence $\lambda \propto P^{1 / 3}$, i.e., $a=0$ and $\alpha=0$ in Eq. (4). The radial mean free path values used are between $\Lambda_{r r}=0.1 \mathrm{AU}$ and 1.0 AU, corresponding to the "Palmer consensus values" (up to $\Lambda_{r r} \sim 0.3 \mathrm{AU}$ ) and to what is often called scatter-free transport. The fitted values of the path length, i.e., $\Delta s=s-s_{0}$ with the distance $s_{0}$ from the source to the observer along the Archimedean spiral subtracted, and the shift of the apparent solar release time, $t_{0}$, relative to the actual release time are plotted 
Table 2. Results of VDA for simulation runs performed to study the onset of SEP events. The event onset criterion $k=0.001$ was used in the VDA. The fitted path lengths are given without error estimates, but the error of the last given digit is less than 5.

\begin{tabular}{|c|c|c|c|c|c|c|c|}
\hline \multirow[t]{2}{*}{ No. } & \multirow[t]{2}{*}{$\tau[\mathrm{h}]$} & \multicolumn{2}{|c|}{$0.13-57 \mathrm{MeV}$} & \multicolumn{2}{|c|}{$1-57 \mathrm{MeV}$} & \multicolumn{2}{|c|}{$0.13-2.5 \mathrm{MeV}$} \\
\hline & & $s[\mathrm{AU}]$ & $t_{0}[\mathrm{~min}]$ & $s[\mathrm{AU}]$ & $t_{0}[\mathrm{~min}]$ & $s[\mathrm{AU}]$ & $t_{0}[\mathrm{~min}]$ \\
\hline 1 & 0 & 2.06 & $4 \pm 6$ & 2.19 & $-11 \pm 2$ & 2.02 & $16 \pm 9$ \\
\hline 2 & 0 & 1.50 & $8 \pm 7$ & 1.58 & $-4 \pm 1$ & 1.46 & $21 \pm 11$ \\
\hline 3 & 0 & 1.35 & $7 \pm 3$ & 1.41 & $-1 \pm 1$ & 1.33 & $15 \pm 4$ \\
\hline 4 & 0 & 1.30 & $5 \pm 2$ & 1.37 & $-2 \pm 1$ & 1.29 & $10 \pm 2$ \\
\hline 5 & 0 & 1.27 & $6 \pm 3$ & 1.33 & $-1.7 \pm 0.4$ & 1.25 & $12 \pm 3$ \\
\hline 6 & 0 & 1.24 & $6 \pm 3$ & 1.30 & $-1.1 \pm 0.9$ & 1.22 & $13 \pm 4$ \\
\hline 7 & 0 & 1.22 & $5 \pm 2$ & 1.27 & $-0.6 \pm 1.0$ & 1.21 & $10 \pm 3$ \\
\hline 8 & 0 & 1.21 & $4 \pm 2$ & 1.25 & $-1.3 \pm 0.2$ & 1.20 & $9 \pm 2$ \\
\hline 1 & 3.7 & 2.20 & $29 \pm 7$ & 2.43 & $2 \pm 1$ & 2.13 & $51 \pm 8$ \\
\hline 2 & 3.7 & 1.60 & $20 \pm 6$ & 1.74 & $2 \pm 1$ & 1.55 & $37 \pm 9$ \\
\hline 3 & 3.7 & 1.44 & $15 \pm 3$ & 1.55 & $2 \pm 1$ & 1.41 & $26 \pm 4$ \\
\hline 4 & 3.7 & 1.37 & $12 \pm 3$ & 1.47 & $0.8 \pm 0.5$ & 1.34 & $20 \pm 3$ \\
\hline 5 & 3.7 & 1.33 & $10 \pm 2$ & 1.41 & $0.6 \pm 0.5$ & 1.31 & $17 \pm 3$ \\
\hline 6 & 3.7 & 1.30 & $10 \pm 3$ & 1.38 & $0.7 \pm 0.8$ & 1.27 & $19 \pm 3$ \\
\hline 7 & 3.7 & 1.27 & $8 \pm 2$ & 1.33 & $0.4 \pm 0.3$ & 1.26 & $14 \pm 2$ \\
\hline 8 & 3.7 & 1.26 & $6 \pm 2$ & 1.31 & $-0.1 \pm 0.3$ & 1.24 & $11 \pm 2$ \\
\hline 9 & 0 & 2.3 & $40 \pm 20$ & 2.64 & $-3 \pm 2$ & 2.2 & $81 \pm 30$ \\
\hline 10 & 0 & 1.59 & $5 \pm 7$ & 1.66 & $-4 \pm 2$ & 1.57 & $14 \pm 11$ \\
\hline 11 & 0 & 1.26 & $6 \pm 3$ & 1.34 & $-3 \pm 1$ & 1.24 & $14 \pm 4$ \\
\hline 9 & 3.7 & 2.4 & $76 \pm 19$ & 3.0 & $11 \pm 6$ & 2.3 & $131 \pm 23$ \\
\hline 10 & 3.7 & 1.69 & $16 \pm 6$ & 1.82 & $-0.6 \pm 0.4$ & 1.65 & $30 \pm 8$ \\
\hline 11 & 3.7 & 1.33 & $8 \pm 2$ & 1.40 & $-0.6 \pm 0.2$ & 1.31 & $14 \pm 3$ \\
\hline 12 & 0 & 1.43 & $53 \pm 12$ & 1.9 & $2 \pm 6$ & 1.31 & $94 \pm 12$ \\
\hline 13 & 0 & 1.23 & $16 \pm 5$ & 1.36 & $1 \pm 2$ & 1.20 & $28 \pm 6$ \\
\hline 14 & 0 & 1.19 & $5 \pm 2$ & 1.24 & $-0.9 \pm 0.3$ & 1.17 & $11 \pm 2$ \\
\hline 12 & 3.7 & 1.51 & $77 \pm 13$ & 2.1 & $13 \pm 5$ & 1.37 & $125 \pm 11$ \\
\hline 13 & 3.7 & 1.27 & $26 \pm 5$ & 1.46 & $5 \pm 2$ & 1.22 & $42 \pm 4$ \\
\hline 14 & 3.7 & 1.21 & $9 \pm 2$ & 1.28 & $1.1 \pm 0.5$ & 1.19 & $15 \pm 1$ \\
\hline 15 & 0 & 1.7 & $78 \pm 20$ & 2.4 & $-3 \pm 11$ & 1.6 & $135 \pm 24$ \\
\hline 16 & 0 & 1.33 & $24 \pm 6$ & 1.56 & $-1 \pm 3$ & 1.27 & $43 \pm 5$ \\
\hline 17 & 0 & 1.22 & $7 \pm 2$ & 1.30 & $-1 \pm 1$ & 1.20 & $14 \pm 2$ \\
\hline 15 & 3.7 & 1.8 & $109 \pm 19$ & 2.7 & $21 \pm 10$ & 1.63 & $175 \pm 15$ \\
\hline 16 & 3.7 & 1.38 & $39 \pm 7$ & 1.67 & $7 \pm 4$ & 1.31 & $63 \pm 5$ \\
\hline 17 & 3.7 & 1.25 & $12 \pm 3$ & 1.35 & $1 \pm 1$ & 1.22 & $21 \pm 2$ \\
\hline
\end{tabular}

in Fig. 2. Typical values for $\Delta s$ as deduced from SEP observations are below $1 \mathrm{AU}$, consistent with our simulations as long as the radial mean free path $\Lambda_{r r} \gtrsim 0.1 \mathrm{AU}$; values smaller than this lead to values of $s$ that are too large to be consistent with the VDA of experimental data. The fitted $s$ values depend on the onset criterion so that increasing $k$ by an order of magnitude increases $\Delta s$ by $30-50 \%$. Thus, there would be a bias toward longer values of the path length in smaller SEP events, if scattering conditions were independent of particle fluxes. For values of $\Delta s<1 \mathrm{AU}$, the particle release time is well estimated (within $10 \mathrm{~min}$ ) with VDA regardless of the value of $k$, whereas with longer path lengths, the value of $t_{0}$ is less accurate.
The effects of the spatial and low-rigidity dependence of the mean free path are studied in Fig. 3. We have used values of $a=30 r_{\odot}$ and $\alpha=1$ along with the standard values of $a=0=\alpha$. The onset criterion here is $k=0.001$. By increasing the low-rigidity mean free path $(\alpha=1)$, we obtain lower values of $\Delta s$, and by decreasing the near-Sun mean free path ( $a=30 r_{\odot}$ ) we obtain higher values of $\Delta s$, as might be expected. By taking both $a=30 r_{\odot}$ and $\alpha=1$, the two effects approximately cancel in the value of $\Delta s$. In the apparent release time, however, both modifications to the scattering conditions work in the same direction, i.e., make the release time seem later than it actually is, if $\Lambda_{r r}$ is small. It should be noted that 

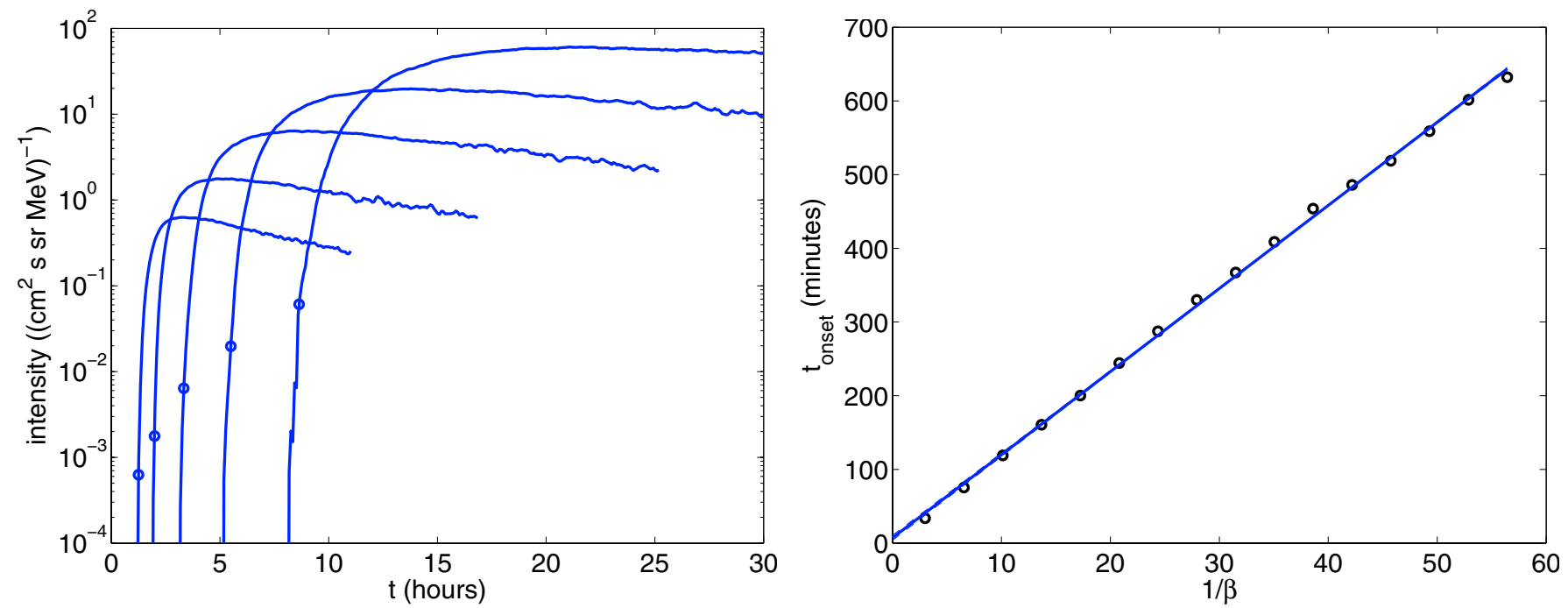

Fig. 1. Proton intensities in five (of 16) energy channels (left panel, from top: $0.19-0.21 \mathrm{MeV}, 0.47-0.60 \mathrm{MeV}, 1.1-1.6 \mathrm{MeV}, 2.5-4.6 \mathrm{MeV}$, and 4.6-11 MeV) and the results of the VDA (right panel) from a simulation with transport parameters $\Lambda_{r r}=0.3 \mathrm{AU}$ and $a=0=\alpha$. The circles in the left panel indicate the channels' onset time (here defined with $k=0.001$ ). In the right panel, channels' onset times (circles) as a function of inverse speed along with a linear fit (line) to the points are given.
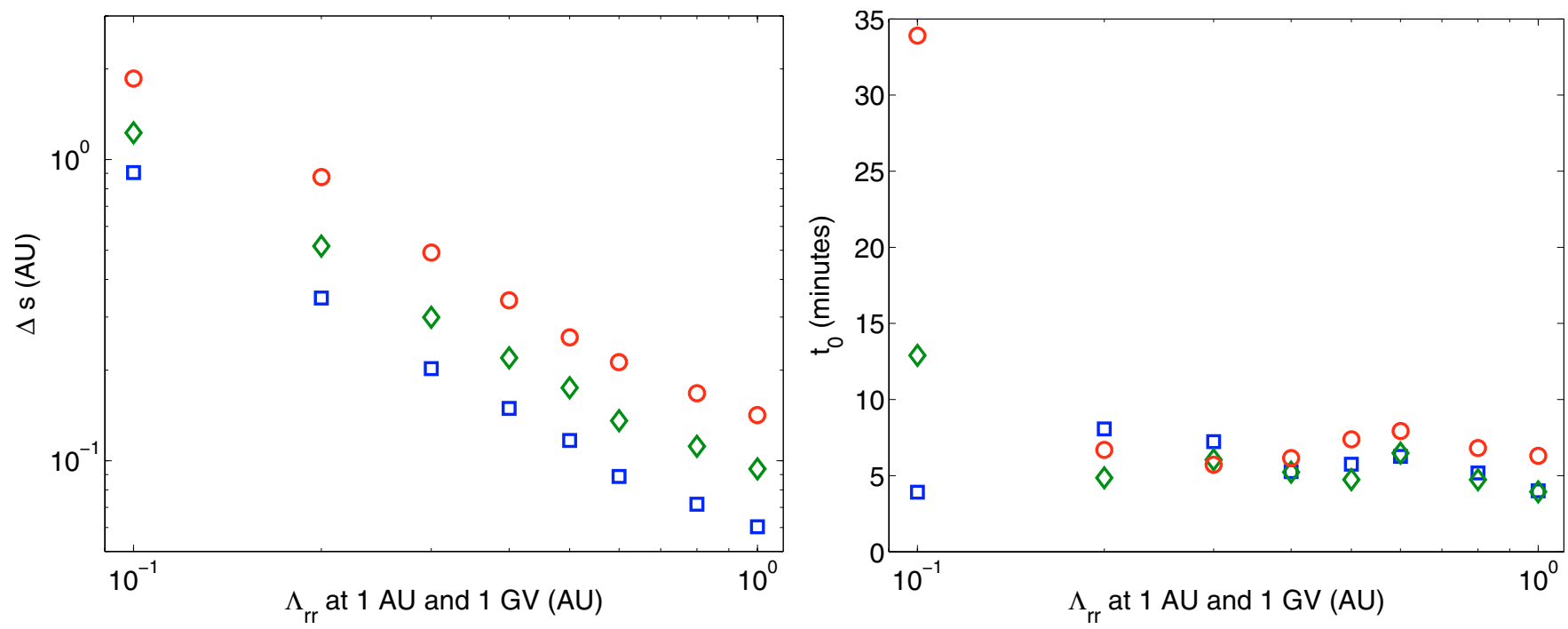

Fig. 2. The fitted path length (left panel) and the time delay (right panel) of the first-arriving protons as a function of the radial proton mean free path. The length of the Archimedean spiral from the source to 1 AU is subtracted from the fitted path length. The event onset criteria of $k=0.1$ (circle), 0.01 (diamond), and 0.001 (box) are used.

the derived VDA parameters for the models with large IP mean free path $\left(\Lambda_{r r} \gtrsim 0.3 \mathrm{AU}\right)$ and small coronal mean free path $\left(a=30 r_{\odot}\right)$ agree well with observations. Scattering conditions in such models give coronal shocks a possibility to rapidly accelerate particles also in small gradual SEP events, which do not yield large intensities of self-generated Alfvén waves (Vainio 2003). Such scattering conditions could result, e.g., from turbulent damping of Alfvén waves in the solar wind (Vainio et al. 2003).

Since the shape of the intensity-time profile during the rise phase determines the channels' onset times, it is necessary also to study the effect of the duration of the particle injection to the VDA. In Fig. 4, we have plotted the VDA fitting parameters as a function of $\Lambda_{r r}$ (for $a=0=\alpha$ and $k=0.001$ ) for three values of the injection duration, $\tau=0,3.7 \mathrm{~h}$, and $9.5 \mathrm{~h}$. We used a stationary source at $1.5 r_{\odot}$, but for a moving, CME-driven shock source these times would correspond to maximum radial injection distances of $0,19 r_{\odot}$, and $49 r_{\odot}$ above the initial distance assuming a radial source speed of $1000 \mathrm{~km} \mathrm{~s}^{-1}$. As can be seen, longer duration of the injection implies longer path length and release-time delay, but the effect seems to saturate somewhat at the largest values of $\tau$. The additional release-time delay varies from a few to about $30 \mathrm{~min}$ in our simulations, thus adding to the uncertainty of the determined onset time.

If SEPs are accelerated by CME-driven shock waves, they are released to the IP medium from a moving source. Although it was seen that the main contribution to the observed time delay comes from the first few hours of the injection, the movement of the source may have an effect on the event onset. Thus, we performed simulations with extended injection from a 

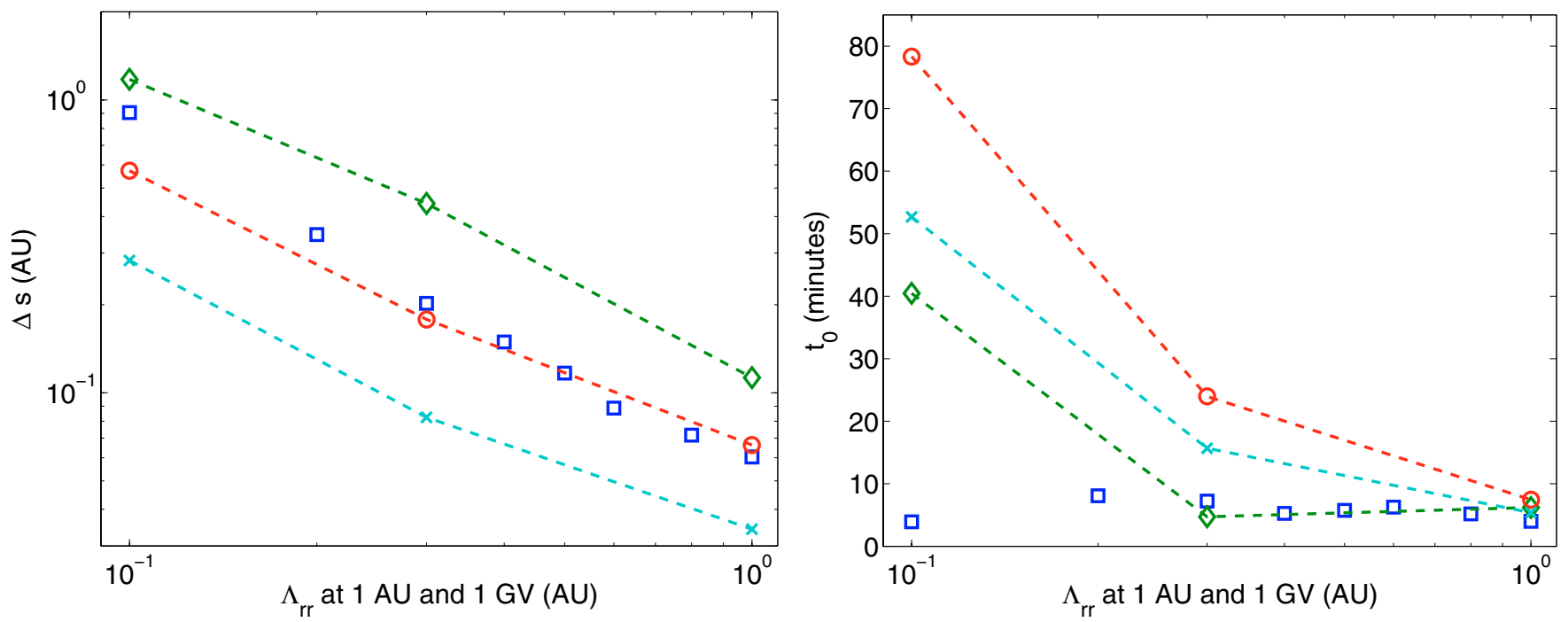

Fig. 3. The fitted path length (left panel) and the time delay (right panel) of the first-arriving protons as a function of the radial proton mean free path. The length of the Archimedean spiral from the source to $1 \mathrm{AU}$ is subtracted from the fitted path length. Other model parameters used are $(\alpha, a)=(0,0)$ (box), $\left(0,30 r_{\odot}\right)$ (diamond), $(1,0)$ (cross), and $\left(1,30 r_{\odot}\right)$ (circle). The event onset criterion is $k=0.001$.
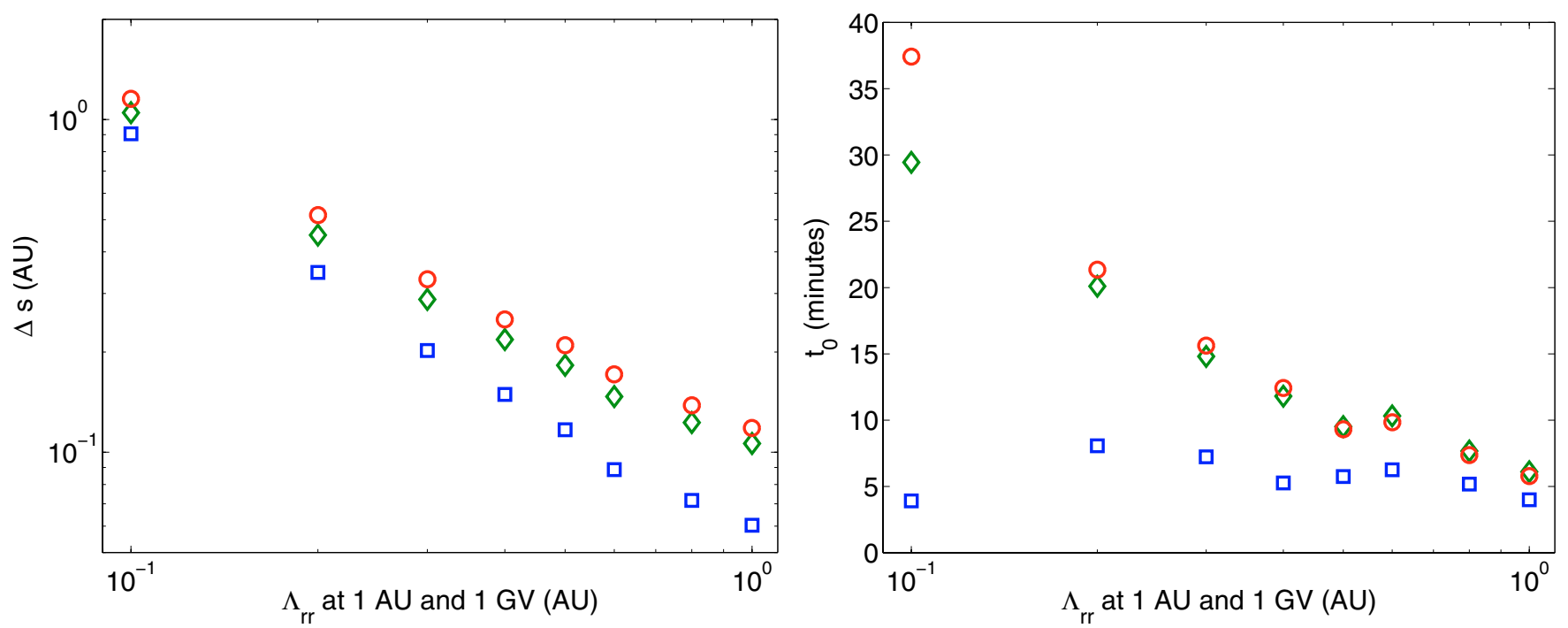

Fig. 4. The fitted path length (left panel) and the time delay (right panel) of the first-arriving protons as a function of the radial proton mean free path. The length of the Archimedean spiral from the source to $1 \mathrm{AU}$ is subtracted from the fitted path length. Other model parameters used are $\alpha=0=a$. The duration of the proton injection is taken to be $\tau=0$ (box), $\tau=3.7 \mathrm{~h}$ (diamond), and $\tau=9.5 \mathrm{~h}$ (circle). The event onset criterion is $k=0.001$.

moving source, mimicking a CME-driven shock. In this case, particles are injected using the source function

$Q_{2}=\frac{\mathrm{d} N}{\mathrm{~d} E} \frac{\mathcal{H}(\tau-t) \mathcal{H}(t)}{\tau} \delta\left\{r-\left(r_{\odot}+V_{\mathrm{S}} t\right)\right\}$

where $V_{\mathrm{S}}=1000 \mathrm{~km} \mathrm{~s}^{-1}$. Temporally constant injection from a source moving at constant speed would correspond to a shock wave continuously accelerating a population of seed particles with a density inversely proportional to the flux-tube crosssectional area. Using the value of $\tau=3.7 \mathrm{~h}$, corresponding to a maximum source distance of $20 r_{\odot}$, and transport parameters $\Lambda_{r r}=0.3 \mathrm{AU}, a=0=\alpha$, we obtain a similar event onset as with a stationary source, as long as $k=0.001$ is used: the difference in the fitted value of $t_{0}$ is within errors of the analysis, and the value of $s$ is slightly increased. Thus, the stationary source approximation is probably not crucial to the analysis. However, with larger values of $k$, a stationary source produces a significantly earlier onset and smaller $s$ than a moving source.

We have also used the simulated data to study the effect of the definition of the event onset on the VDA results. In an experimental situation, SEP event onset may occur on a variety of pre-event conditions. This can obviously have an effect on the results of the onset analysis. As examples, we consider the following scenarios: (i) an event starting on a quiet-time background with proton intensities of

$I_{\mathrm{q} . \mathrm{t} .}(E)=I_{1} \frac{\left(E_{1}+E\right)^{3}}{8 E_{1} E^{2}}$ 

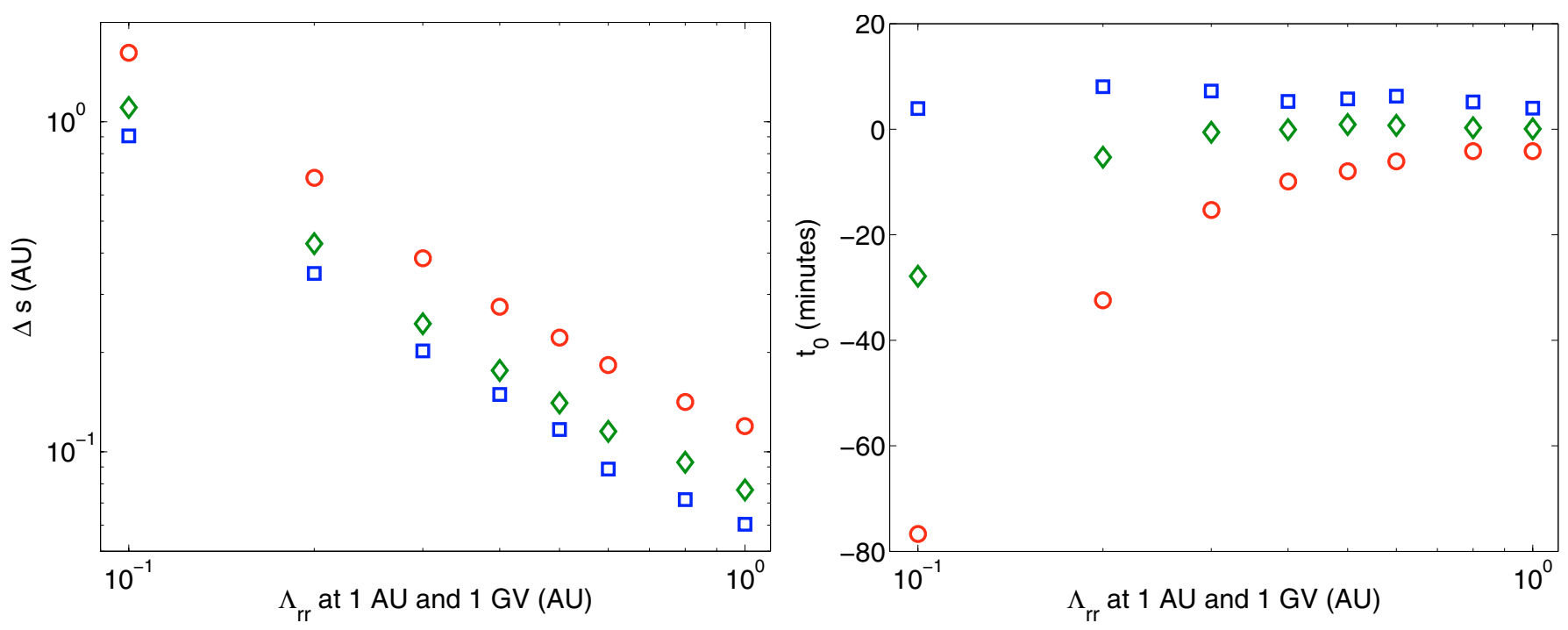

Fig. 5. The fitted path length (left panel) and the time delay (right panel) of the first-arriving protons as a function of the radial proton mean free path. The length of the Archimedean spiral from the source to $1 \mathrm{AU}$ is subtracted from the fitted path length. Other model parameters used are $\alpha=0=a$. The event onset criterion is $k=0.001$ (box) in one of the analyses, and the other two are based on quiet-time (diamond) and previous-event (circle) background intensities.

with $I_{1}=I_{\text {q.t. }}\left(E_{1}\right)=2 \times 10^{-4}$ protons $\left(\mathrm{cm}^{2} \mathrm{~s} \mathrm{sr} \mathrm{MeV}\right)^{-1}$ and $E_{1}=20 \mathrm{MeV}$, and (ii) an event starting on the tail of a previous one with proton intensities of

$I(E, t)=I_{\text {q.t. }}(E)+I_{2}\left(\frac{\mathrm{MeV}}{E}\right)^{3} \exp \left(-\frac{t}{t_{\mathrm{d}}}\right)$

with $I_{2}=0.02$ protons $\left(\mathrm{cm}^{2} \mathrm{~s} \mathrm{sr} \mathrm{MeV}\right)^{-1}, E_{2}=1 \mathrm{MeV}$, and $t_{\mathrm{d}}=24 \mathrm{~h}$. The quiet-time background is clearly above typical physical proton intensities in the IP medium, because the background level in real SEP instruments usually consists of instrumental noise in addition to the quiet-time particle fluxes. This choice of the background intensities is, of course, arbitrary in the sense that no particular instrument or previous SEP event is modeled. Using these background intensity levels, the event onset is defined as the first time the proton intensity increases over 1.5 times the momentary background level. The results for the VDA are plotted in Fig. 5. Since the studied background spectra are softer than the time-of-maximum spectrum produced by our injection spectrum, the pre-event background acts here to delay the onset of the low-energy channels more than high-energy channels. Especially for small values of the mean free path, background intensity levels have a clear effect on the VDA parameters, especially the release-time delay. For a soft pre-event background prevailing, e.g., after the passage of IP shock waves, a subsequent hard particle event may show apparent release times which are tens of minutes earlier than the actual particle release time at the Sun. The values of the apparent release times are, of course, dependent on the contrast between the background and time-of-maximum intensities.

Finally, we study the effect of the applied energy range on the results of the VDA. In Fig. 6, we plot the fitted apparent time delay vs. the fitted path length increase for three energy ranges, i.e., $0.13-2.5 \mathrm{MeV}, 1-57 \mathrm{MeV}$, and $0.13-57 \mathrm{MeV}$, corresponding to data from instruments operating at low and high-energies, and their combination. The "standard scenario"

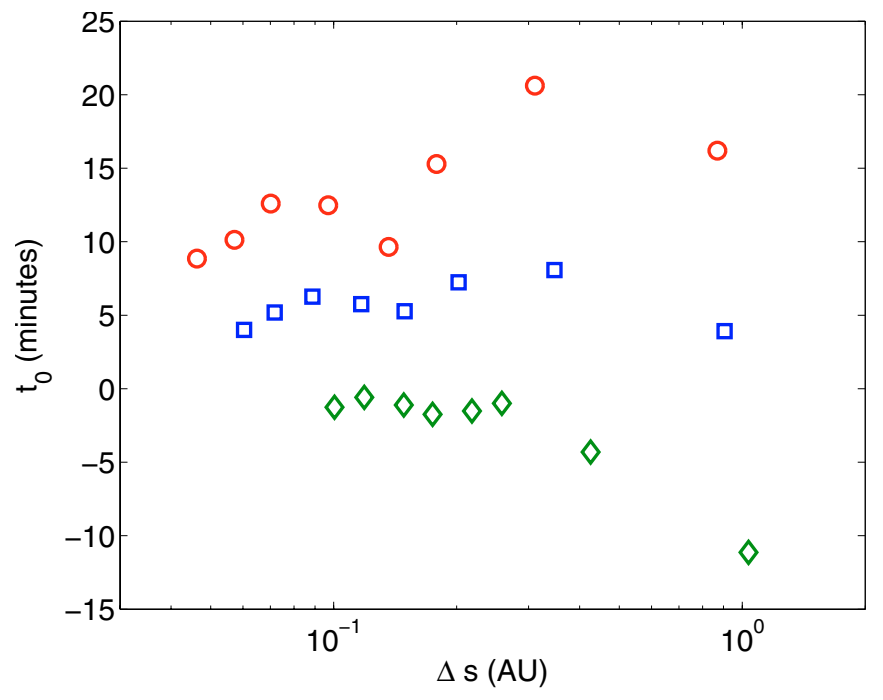

Fig. 6. The fitted time delay vs. path length of the first-arriving protons. The length of the Archimedean spiral from the source to $1 \mathrm{AU}$ is subtracted from the fitted path length. Other model parameters used are $\alpha=0=a$, and individual points correspond to different values of $\Lambda_{r r}$. The event onset criterion is $k=0.001$. The applied proton energy range is $0.13-57 \mathrm{MeV}$ (box), $0.13-2.5 \mathrm{MeV}$ (circle), and 1-57 (diamond).

$\left(\alpha=a=\tau=0, \Lambda_{r r} \in[0.1,1.0] \mathrm{AU}, k=0.001\right)$ is used in each case. At high energies, the analysis produces onset times that are from about 10 to 30 min earlier than values obtained at low energies for the same simulation parameters or at constant $\Delta s$. For some other simulation parameters, low-energy VDA produces earlier release times than high-energy analysis. Thus, rather the scatter than the actual values of the data points are important in this figure. 


\section{Conclusions}

We have performed a study of SEP event onset based on simulated data. The purpose of this paper is, on the one hand, to find out whether simple VDA can give reliable results for particle release times at the Sun and, on the other hand, to see if the VDA results can give some clues about the coronal and IP transport conditions. As a result of a detailed parametric study of event onset using simulated data sets, we arrive at the following conclusions:

- Typically observed values of the apparent path length traversed by protons after their release from the coronal acceleration site $(s \sim 1-2 \mathrm{AU})$ imply that the IP mean free path is during most of the events above $\Lambda_{r r} \gtrsim 0.1 \mathrm{AU}$ (at $1 \mathrm{GV}$ rigidity). Values of the mean free path below this limit should yield large values of $s$, and such events should be easily identified from observational data. Timing obtained for such events using VDA is uncertain.

- Models with typical IP mean free path $\left(\Lambda_{r r} \gtrsim 0.3 \mathrm{AU}\right)$ combined with a small coronal mean free path $\left(\lambda \ll r_{\odot}\right.$ at $1 \mathrm{GV}$ rigidity) produce apparent path lengths consistent with observations. Such scattering conditions enable coronal shock waves to accelerate particles efficiently without the need to introduce self-generated Alfvén waves or quasiperpendicular shock geometry.

- Apparent release times obtained from simulated data correspond rather well (within a few tens of minutes) to the actual start time of the injection for large values of the mean free path $\Lambda_{r r}>0.3 \mathrm{AU}$. Such events can be recognized from observational data from values of $s \lesssim 1.5 \mathrm{AU}$.

- Pre-event flux conditions have a notable effect on the results of VDA. Events with a small contrast between the time-of-maximum and pre-event background intensity levels and/or a large difference between the respective spectral indices may yield poor results for the particle release times obtained using VDA.
- The relatively large scatter in values of $s$ obtained for typical IP scattering conditions implies that RTA, which applies predefined path lengths close to $s=1 \mathrm{AU}$, may yield poor results for the injection scenario.

Acknowledgements. The authors thank Timo Laitinen for suggesting the idea to study SEP event onsets using simulated data.

\section{References}

Bieber, J. W., Matthaeus, W. H., Smith, C. W., et al. 1994, ApJ, 420, 294

Kahler, S. W. 1994, ApJ, 428, 837

Klein, K.-L., Chupp, E. L., Trottet, G., et al. 1999, A\&A, 348, 271

Kocharov, L., Vainio, R., Kovaltsov, G. A., \& Torsti, J. 1998, Sol. Phys., 182, 195

Krucker, S., \& Lin, R. P. 2000, ApJ, 542, L61

Mewaldt, R. A., Cohen, C. M. S., Haggerty, D. K., et al. 2003, Proc. 28th Internat. Cosmic Ray Conf., SH 1, 3313

Palmer, I. D. 1982, Rev. Geophys. Space Phys., 20, 335

Posner, A., \& Kunow, H. 2003, Proc. 28th Internat. Cosmic Ray Conf., SH 1, 3309

Reames, D. V. 1999, Space Sci. Rev., 90, 413

Ruffolo, D. 1995, ApJ, 442, 861

Schlickeiser, R., \& Achatz, U. 1993, J. Plasma Phys., 49, 63

Torsti, J., Anttila, A., Kocharov, L., et al. 1998, Geophys. Res. Lett., 25,2525

Tylka, A. J., Cohen, C. M. S., Dietrich, W. F., et al. 2003, Proc. 28th Int. Cosmic Ray Conf., SH 1, 3313

Vainio, R. 2000, ApJS, 131, 738

Vainio, R. 2003, A\&A, 406, 735

Vainio, R., \& Khan, J. I. 2004, ApJ, 600, 451

Vainio, R., Kocharov, L., \& Laitinen, T. 2000, ApJ, 528, 1015

Vainio, R., Laitinen, T., \& Fichtner, H. 2003, A\&A, 407, 713

Zhang, M., Jokipii, J. R., \& McKibben, R. B. 2003a, ApJ, 595, 493

Zhang, M., McKibben, R. B., Lopate, C., et al. 2003b, J. Geophys. Res., 108(A4), SH4-1 